\title{
Patient with Lobular Carcinoma of the Breast and Activating AKT1 E17K Variant
}

\author{
Harsha Trivedi ${ }^{1}$, Omar Hamdani ${ }^{2}$, Brittani Thomas ${ }^{1}$, James Richard ${ }^{3}$, Kunal Shah ${ }^{2}$, Kira Raskina ${ }^{2}$, Liang Zhang ${ }^{2}$, \\ Audrey Madigan ${ }^{2}$, Marshall Fox ${ }^{2}$, Seth Wander ${ }^{2}{ }^{4}$, Matthew J Frigault ${ }^{2}{ }^{4}$, Brian Alexander ${ }^{2}$, Gordan Srkalovic ${ }^{1}$ \\ ${ }^{1}$ Herbert-Herman Cancer Center, E. W. Sparrow Hospital, Lansing, MI, USA, ${ }^{2}$ FoundationMedicine, Cambridge, MA, USA, \\ ${ }^{3}$ Department of Pathology and Laboratory Medicine, Sparrow Hospital System, Lansing, MI, USA, ${ }^{4}$ Massachusetts General \\ Hospital, Boston, MS, USA
}

Correspondence: gordan.srkalovic@sparrow.org; Tel.: + 1517364 2182; Fax.: + 15173642886

Received: 24 February 2021; Accepted: 25 February 2021

\begin{abstract}
Objective. To present the characteristics of the AKT1E117K gene variant and a description of the clinical application in a patient with metastatic breast cancer. Results. 63 y/o woman with Stage IV Invasive lobular carcinoma at diagnosis was treated with Palbociclib and aromatase inhibitors (AI). At progression, tissue was sent for comprehensive genomic profiling to Foundation Medicine (FM) which revealed AKT1E17K mutation. In lieu of available clinical data within the patient's tumor type (HR+ HER2- breast cancer), extrapolated data from the Flatiron Health-FM (FH-FMI) Clinico-genomic Database (CGDB) was discussed at our Molecular Tumor Board (MTB). After multidisciplinary discussion, the consensus recommendation was to start treatment with the combination of mTOR inhibitor everolimus, and AI, exemestane. Patient tolerated treatment without major side effects. By the second clinical visit the patient's breast showed signs of improvement. PET/CT showed diminished left axillary uptake, decreased right paratracheal lymph node PET avidity, and stable bone disease consistent with a partial response. The most recent office visit in January 2021, breast exam revealed a normal-appearing skin with only faint erythema. All other skin lesions have resolved. Although, the role of AKT1 variant described here is not well defined and therapeutic significance of M-Tor inhibitors not established in metastatic breast cancers, comprehensive approach to this case unraveled new and successful therapeutic option in this patient. Conclusion. This demonstrates that applying available Precision Medicine tools like MTB and real world data sets from patient populations with similar clinical and genomic profiles may provide more options for treatment.
\end{abstract}

Key Words: AKT1 - Breast Cancer - Molecular Tumor Board (MTB) - CGDB - Comprehensive Genomic Data Base.

\section{Introduction}

Invasive lobular carcinoma (ILC) is the most common of the breast cancer special types, accounting for up to $15 \%$ of all breast cancer cases. ILCs are noted for their lack of E-cadherin function, which results in non-cohesive growth pattern, with the knowledge from genomic profiling now there is huge amount of new data, from the genomic landscape of ILC and in particular somatic alterations associated with therapy resistance, and the evolution of several potential therapeutic avenues. Many targeted and chemotherapy options are being evaluated.

Here we present a case of ILC treated at our cancer center based on the precision medicine tools. The information provided by genomic profile is used clinically to guide treatments decisions for approved targeted therapies and in clinical trials. In this case none of that could be used. We found an effective treatment for this patient based on the MTB discussion and the real world data of the AKT1E17K variant and AKT1 WT breast cancer patients.

\section{Results}

\section{Clinical Presentation}

A 63-year-old pleasant woman with no major past medical history presented in January 2019 with a palpable left breast mass. On physical exam, besides the breast mass, the patient had palpable lymph nodes in the left axilla. She was referred for 


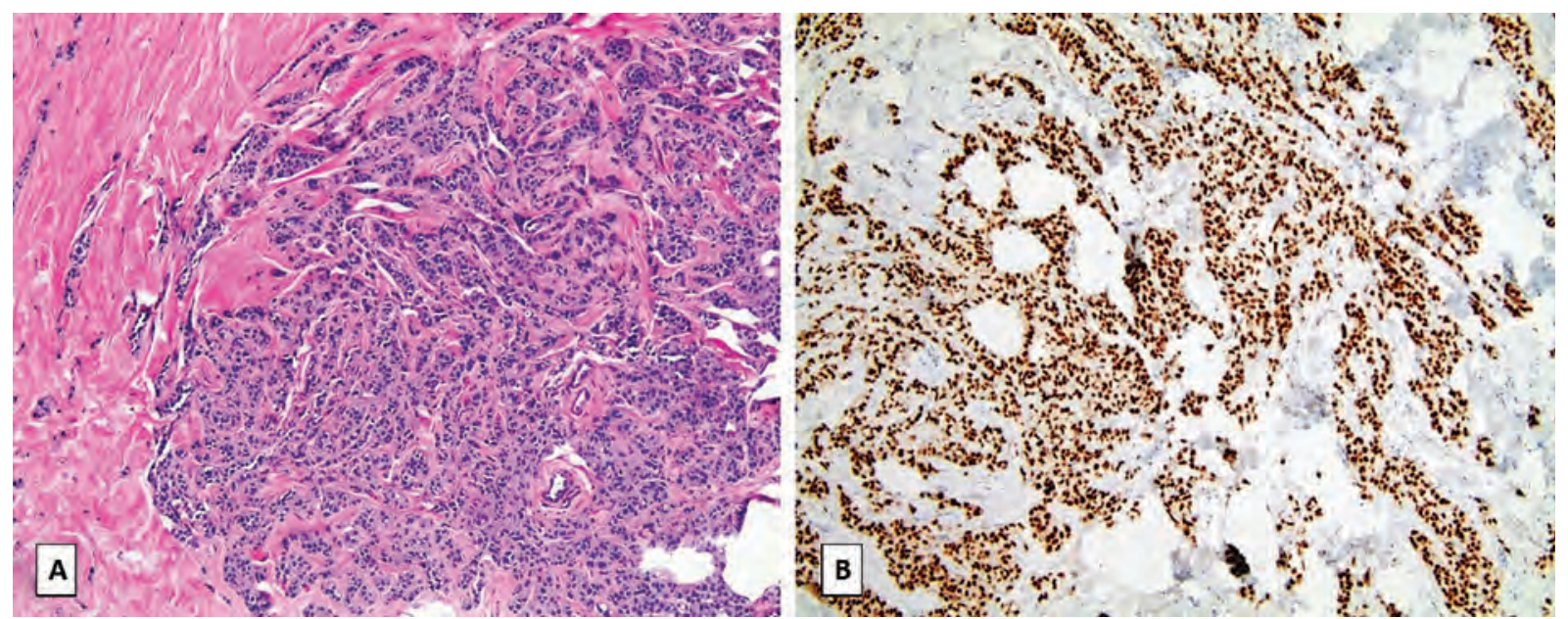

Figure 1. (A): A core needle biopsy was showing invasive lobular carcinoma infiltrating the fibrous tissue of the breast as single cells, cords, or linear strands of neoplastic cells, most often found in the desmoplastic breast stroma (Hematoxylin and Eosin stain); (B): Immunohistochemistry (IHC) of invasive lobular carcinoma patient showing diffuse and strong estrogen receptor (ER) expression. The patient's sample was PR negative and HER2 negative. Ki-67 was positive in $~ 50 \%$ cancer cells.

diagnostic mammography and ultrasound, which revealed a diffuse nonhomogeneous density at the site of palpable finding. A core needle biopsy was performed, and pathology revealed classic invasive lobular carcinoma infiltrating the fibrous tissue of the breast as single cells and in cords or linear strands of cells, most often found in the breast fibrous stroma (Figure 1A).

The infiltrating cells are monotonous, small in size, and possess round nuclear contours. This pattern of invasion can at times encircle benign ducts in a concentric or "targetoid" fashion. These cells can be seen infiltrating together, as in the image (Figure 1B) or in a rather insidious fashion separated by the fibrous stroma of the breast, presenting a challenge to find for even an experienced pathologist. This is especially challenging when the amount of tumor present is limited, as in needle biopsy material. The classic invasive lobular carcinoma typically expresses estrogen (ER) and progesterone receptors (PR) and is negative for HER2 expression.In this case, the cancer cells were ER-positive while PR and HER2 receptors were negative. The proliferation rate was $\sim 50 \%$ (measured by Ki-67).

On February 5, 2019, the patient underwent a PET/CT scan, which revealed diffuse skeletal metastases and extensive left axillary lymphadenopa- thy (LADP) extending into the pectoralis minor muscle and the thoracic outlet. After this workup, the patient was staged as Stage IV Invasive Lobular Carcinoma of the left breast cT3N3M1, grade 2. The patient has an indeterminate right middle lobe pulmonary nodule, which was slightly fluorodeoxyglucose (FDG) avid. Genetic testing by the Invitae Panel was done on 5/7/19 and was negative for any deleterious mutations. At that time patient was treated with a combination of CDK4/6 inhibitor palbociclib and aromatase inhibitor (AI) letrozole. Zoledronic acid was also initiated as bone targeting treatment. The patient tolerated treatment with the support of growth factors for low counts and palbociclib dose reduction to $100 \mathrm{mg}$ from May 2019. She tolerated further treatments without any major side effects. PET/CT scan in August 2019 showed significant positive interval response with resolution of hypermetabolic thoracic outlet and left axillary LADP, as well as decreasing left breast activity and decreasing FDG avidity of several osseous lesions. Scan in January 1/31/20 was reported as no evidence of disease (NED).

However, on a follow-up visit in February 2020, the patient complained that her left breast was feeling "heavier". She denied pain and struggled to describe the change. On physical exam, the patient had an erythematous rash involving 
the medial half of the breast. The breast was not tender, and there were no palpable masses. Biopsy of the involved skin was done, and pathology confirmed lobular carcinoma with no changes in receptor status. A repeated PET/CT scan in March 2020 showed increased left anterior breast skin thickening, a mildly increased uptake within the left axillary lymph nodes, and an increased overall number and extent of osseous metastases. The patient also had several small skin lesions on the neck and chest. Biopsy of the left neck lesion confirmed metastatic lobular carcinoma with the same characteristics as previously reported. The patient continued on palbociclib, while the treatment with letrozole was changed to the ER antagonist, fulvestrant. She enjoyed a clinical response from April to August 2020. However, on her office follow-up in August, the left breast was again increasingly erythematous and swollen. The erythema involved about three-quarters of the breast, and PET/CT scan confirmed increasing osseous metastatic burden.

\section{Genomic Analysis}

To determine whether the patient was a candidate for the use of targeted therapy based on her tumor genomics, tissue from her neck skin biopsy was sent for Foundation One CDx (F1CDx) solid biopsy CGP and evaluation of PD-L1 receptor. F1CDx is a hybrid-capture-based CGP assay that baits exonic regions of 324 genes and selects intronic regions for rearrangements (FMI2021). The test can detect gene alterations (GA) in the form of short variants (SV), rearrangements (RE), and copy number alterations (CNA), including amplifications / homozygous deletions. The patient's CGP results showed detection of a previouslycharacterized and predicted activating AKT1 E17K mutation. We characterize this gene variant and attempt to assign its clinical significance below.

$A K T 1$ is an intracellular serine/threonine kinase that can phosphorylate and activate the serine/threonine kinase mTORi (1). Upon activation, the mTORi complex can stimulate cell proliferation and growth through a variety of oncogenic mecha- nisms. AKT1 is situated downstream of PI3K and upstream of mTORi in the PI3K-mTOR signaling pathway, which suggests inhibition of AKT1 or downstream signaling components could be an effective treatment in $A K T 1$ altered cancers. It is important to note that the AKT1 kinase phosphorylates other proteins, which may have inhibitory effects on cell growth. This diverse biology suggests that AKT1 influences several mechanisms spanning both oncogenic and anti-oncogenic effects (2-5).

AKT1 E17K variant has been extensively characterized preclinically in a variety of cancer cell types and model systems (6-11). AKT1 E17K is a hotspot mutation occurring at the N-terminal of the $A K T 1$ protein $(1,12)$. In breast cancer cell line models, ectopic expression of $A K T 1$ E17K leads to increased phosphorylation of AKT1 target genes, inhibition of apoptosis, increased colony formation, and increased tumor growth in mouse xenograft models $(6,7)$. Furthermore, AKT1 E17K mutant breast cancer preclinical models demonstrate sensitivity to inhibition of the mTORi pathway using several targeted therapy agents.

AKT1 mutations occur in $4 \%$ of breast cancer patients, and $A K T 1$ E17K mutations account for $\sim 80 \%$ of those AKT1 mutations (COSMIC database 2021). AKT1 is still in an early stage clinical development as a biomarker for mTOR pathway targeted therapy, but clinical trials are underway (13-17). Interestingly, oncogenic properties that AKT1 E17K cancer cells displayed in a preclinical setting have been recapitulated in a clinical setting. The mTOR pathway activity that has been demonstrated in preclinical AKT1 E17K mutant breast cancer models has also been shown in breast cancer patient samples through pharmacodynamic analysis (i.e., mTORC1 activation and target gene activation). Clinical study also suggests that AKT1 $E 17 \mathrm{~K}$ breast cancer patients may spend longer time on mTORi therapy (i.e., everolimus) than AKT1 wild- type (WT) patients (16), and early phase clinical data suggests that $A K T 1$ E17K mutant ER+ breast cancer patients may benefit from AKT inhibitors such as capivasertib $(15,17)$. 
Collectively, these data indicate that inhibition of mTOR pathway components may be a treatment option for $A K T 1 E 17 K$ mutant breast cancer patients.

\section{Application of Precision Medicine Tools}

This report was discussed at our Molecular Tumor Board (MTB) joint activity between Sparrow Herbert-Herman Cancer Center and Foundation Medicine Inc. (FMI) (18). As an educational program, the goal of an FMI MTB program is to discuss the targetability of genomic alterations that are identified by FMI CGP. The focus of discussion during the MTB was whether mTORi pathway inhibition is a viable treatment option for $A K T 1$ $E 17 \mathrm{~K}$ altered breast cancer patients. As noted earlier, the available clinical data for AKT1 targetability is still accumulating. In lieu of clinical data within the patient's tumor type (HR+ HER2- breast cancer), extrapolated data was discussed. However, this is not always appropriate or useful to ascertain treatment options for the MTB patient. One tool available to FMI cancer researchers is the Flatiron Health-Foundation Medicine (FH-FMI) Clinicgenomic Database (CGDB). Retrospective longitudinal clinical data were derived from electronic health record (EHR) data, comprising patient-level structured and unstructured data, curated via technology-enabled abstraction, and were linked to genomic data derived from FMI comprehensive genomic profiling (CGP) tests in the FH-FMI CGDB by de- identified, deterministic matching (19-22).

To better understand how the present patient may respond to $\mathrm{mTORi}$ treatment, a real-world data cohort consisting of HR+/HER2- breast cancer patients with $A K T 1$ E17K alteration were analyzed for treatment use by a line of therapy from the CGDB. Clinical characteristics and treatment history were obtained via technology-enabled abstraction of clinician notes and radiology/pathology reports for $3155 \mathrm{HR}+/ \mathrm{HER} 2-\mathrm{BC}$ patients. AKT1 E17K mutations were found in 143 patients while2964 patients had AKT1 WT. Thirty-one AKT1 E17K and 627 of AKT1 WT patients received mTORi (Figure 2).
7681 patients with BC in CGDB Q4 2020 $\downarrow$

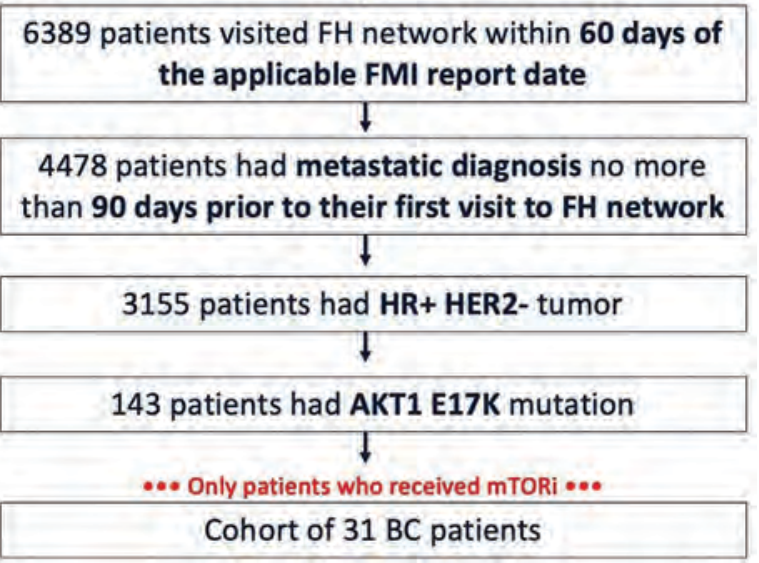

Figure 2. CGDB Cohort of HR+/HER2- Breast Cancer Patients with AKT1 E17K Mutation that Received mTORi.

Time to next treatment (TTNT) was also estimated with Kaplan-Meier analysis and hazard ratios from Cox proportional hazards models. Propensity-score matching (PSM) was used to account for the characteristics that predict receiving of the treatment. The cohort of patients with AKT1 E17K mutant BC receiving mTORi did not significantly differ on major demographic, clini$\mathrm{cal}$, and genetic characteristics from the $A K T 1 \mathrm{WT}$ cohort receiving the same treatment (Table 1). To compare TTNT on mTORi in the two cohorts, we matched the AKT1 E17K patients to AKT1 WT patients on age, tumor type, ECOG, TMB, and mTORi line using PSM.The TTNT on mTORi of $A K T 1 E 17 K$ vs. AKT1 WT patients was 6.5 months (95\% CI 4.6 - n/a) and 8.7 months (95\%CI 6.4 na), respectively. The relative efficacy of receiving mTORi was not significantly higher in AKT1 E17K group ( $\mathrm{HR}=1.2$ [95\%CI: $0.6-2.3], \mathrm{P}=0.6$ ). The only characteristic by which the AKT1 E17K cohorts receiving chemo vs. mTORi were different was the line of treatment (Table 2). A noticeably higher percentage of patients received chemotherapy in earlier lines $(79.7 \%$ in lines $1-2)$, while mTORi was chosen in later lines of treatment (77.4\% in lines 3+). To compare TTNT on mTORi vs. on chemotherapy in these cohorts, we matched the patients who received chemotherapy to the patients who received mTORi on age, tumor 
Table 1. Demographic Information for HR+ HER2- Breast Cancer Patients with or without AKT1 E17K Mutation that Received mTORi from the CGDB

\begin{tabular}{|c|c|c|c|c|}
\hline Demographics & $A K T 1 E 17 K(\mathrm{~N}=31)$ & AKT1 WT $(\mathrm{N}=767)$ & P-value & $\mathrm{P}$ adjusted (FDR) \\
\hline Age at Dx, yrs, Median (IQR) & $52.0(46.0,58.0)$ & $54.0(46.0,62.0)$ & 0.315 & 0.999 \\
\hline Female & $31(100.0 \%)$ & $760(99.1 \%)$ & 0.593 & 0.999 \\
\hline Race & & & 0.808 & 0.999 \\
\hline Asian & $1(3.2 \%)$ & $14(1.8 \%)$ & - & \\
\hline Black or African American & $1(3.2 \%)$ & $45(5.9 \%)$ & - & - \\
\hline Hispanic or Latino & $0(0.0 \%)$ & $2(0.3 \%)$ & - & - \\
\hline White & $25(80.6 \%)$ & $541(70.5 \%)$ & - & - \\
\hline Other Race & $3(9.7 \%)$ & $134(17.5 \%)$ & - & - \\
\hline Not documented & $1(3.2 \%)$ & $31(4.0 \%)$ & - & - \\
\hline Stage at Dx & & & 0.11 & 0.99 \\
\hline $\mathrm{I}-\mathrm{II}$ & $18(58.1 \%)$ & 305 (39.8\%) & - & - \\
\hline III-IV & $12(38.7 \%)$ & $399(52.0 \%)$ & - & - \\
\hline Not documented & $1(3.2 \%)$ & $63(8.2 \%)$ & - & - \\
\hline Tumor Grade & & & 0.874 & 0.999 \\
\hline Grade 1 & $1(3.2 \%)$ & $40(5.2 \%)$ & - & - \\
\hline Grade 2 & $10(32.3 \%)$ & 205 (26.7\%) & - & - \\
\hline Grade 3 & $8(25.8 \%)$ & $193(25.2 \%)$ & - & - \\
\hline Not documented & $12(38.7 \%)$ & $329(42.9 \%)$ & - & - \\
\hline Tumor Type & & & 0.954 & 0.999 \\
\hline IDC & $10(32.3 \%)$ & $255(33.2 \%)$ & - & - \\
\hline ILC & $2(6.5 \%)$ & $59(7.7 \%)$ & - & - \\
\hline Other & $19(61.3 \%)$ & $453(59.1 \%)$ & - & - \\
\hline Community practice & $28(90.3 \%)$ & $708(92.3 \%)$ & 0.686 & 0.999 \\
\hline MFI, yrs, Median (IQR) & $3.7(0.2,7.4)$ & $3.0(0.0,7.25)$ & 0.656 & 0.999 \\
\hline Solid biopsy & $27(87.1 \%)$ & $668(87.1 \%)$ & 0.999 & 0.999 \\
\hline Metastases sites & & & 0.876 & 0.999 \\
\hline Bone-only & $3(9.7 \%)$ & $62(8.1 \%)$ & - & - \\
\hline CNS & $8(25.8 \%)$ & 177 (23.1\%) & - & - \\
\hline Visceral & $20(64.5 \%)$ & $527(68.8 \%)$ & - & - \\
\hline ECOG & & & 0.0353 & 0.635 \\
\hline 1 & $7(31.8 \%)$ & $230(47.3 \%)$ & - & - \\
\hline 2 & $2(9.1 \%)$ & $53(10.9 \%)$ & - & - \\
\hline 3 & $2(9.1 \%)$ & $7(1.4 \%)$ & - & - \\
\hline PD-L1 status & & & 0.388 & 0.999 \\
\hline Negative & $2(6.5 \%)$ & $86(11.2 \%)$ & - & - \\
\hline Positive & $0(0.0 \%)$ & $26(3.4 \%)$ & - & - \\
\hline Not documented & $29(93.5 \%)$ & $655(85.4 \%)$ & - & - \\
\hline TMB (RUO), muts/mB, Median (IQR) & $2.6(1.3,4.5)$ & $2.6(1.3,5.2)$ & 0.541 & 0.999 \\
\hline MSI (RAW) & & & 0.933 & 0.999 \\
\hline MSI-H & $0(0.0 \%)$ & $2(0.3 \%)$ & - & - \\
\hline MSI-I & $0(0.0 \%)$ & $4(0.5 \%)$ & - & - \\
\hline MSS & $21(67.7 \%)$ & $543(70.8 \%)$ & - & - \\
\hline Not documented & $10(32.3 \%)$ & $218(28.4 \%)$ & - & - \\
\hline mTORi & & & 0.727 & 0.999 \\
\hline Everolimus & $31(100.0 \%)$ & 764 (99.6\%) & - & - \\
\hline Temsirolimus & $0(0.0 \%)$ & $3(0.4 \%)$ & - & - \\
\hline Start Date, Median (Range) & 2017-09-18 (2013-03-01 - 2020-06-09) & 2017-08-22 (2011-12-29 - 2020-09-23) & 0.84 & 0.999 \\
\hline mTORi line & & & 0.31 & 0.999 \\
\hline $1-2$ & $7(22.6 \%)$ & $239(31.2 \%)$ & - & - \\
\hline $3+$ & $24(77.4 \%)$ & $528(68.8 \%)$ & - & - \\
\hline Deceased & $22(71.0 \%)$ & $492(64.1 \%)$ & 0.437 & 0.999 \\
\hline
\end{tabular}

MFI=Metastasis-free interval. 
Acta Medica Academica 2021;50(1):209-217

Table 2. Demographic Information for HR+ HER2- Breast Cancer Patients with AKT1 E17K Mutation that Received mTORi vs. Chemotherapy from the CGDB

\begin{tabular}{|c|c|c|c|c|c|}
\hline Demographics & Chemotherapy $(\mathrm{N}=74)$ & mTORi $(\mathrm{N}=31)$ & P-value & $P$ adjusted (FDR) & * \\
\hline Age at Dx, yrs, Median (IQR) & $55.0(46.0 ; 62.0)$ & $52.0(46.0 ; 58.0)$ & 0.366 & 0.93 & \\
\hline Female & $74(100.0 \%)$ & $31(100.0 \%)$ & 0.932 & 0.932 & \\
\hline Race & & & 0.932 & 0.932 & \\
\hline Asian & $2(2.7 \%)$ & $1(3.2 \%)$ & - & & \\
\hline Black or African American & $2(2.7 \%)$ & $1(3.2 \%)$ & - & - & \\
\hline White & $55(74.3 \%)$ & $25(80.6 \%)$ & - & - & \\
\hline Other Race & $12(16.2 \%)$ & $3(9.7 \%)$ & - & - & \\
\hline Not documented & $3(4.1 \%)$ & $1(3.2 \%)$ & - & - & \\
\hline Stage at Dx & & & 0.678 & 0.93 & \\
\hline I-II & $36(48.6 \%)$ & $18(58.1 \%)$ & - & - & \\
\hline III-IV & $35(47.3 \%)$ & $12(38.7 \%)$ & - & - & \\
\hline Not documented & $3(4.1 \%)$ & $1(3.2 \%)$ & - & - & \\
\hline Tumor Grade & & & 0.652 & 0.93 & \\
\hline Grade 1 & $5(6.8 \%)$ & $1(3.2 \%)$ & - & - & \\
\hline Grade 2 & $30(40.5 \%)$ & $10(32.3 \%)$ & - & - & \\
\hline Grade 3 & $18(24.3 \%)$ & $8(25.8 \%)$ & - & - & \\
\hline Not documented & $21(28.4 \%)$ & $12(38.7 \%)$ & - & - & \\
\hline Tumor Type & & & 0.672 & 0.93 & \\
\hline IDC & $19(25.7 \%)$ & $10(32.3 \%)$ & - & - & \\
\hline ILC & $8(10.8 \%)$ & $2(6.5 \%)$ & - & - & \\
\hline Other & $47(63.5 \%)$ & $19(61.3 \%)$ & - & - & \\
\hline Community practice & $68(91.9 \%)$ & $28(90.3 \%)$ & 0.793 & 0.932 & \\
\hline MFI, yrs, Median (IQR) & $2.9(1.3,7.1)$ & $3.7(0.2,7.4)$ & 0.534 & 0.93 & \\
\hline Solid biopsy & $65(87.8 \%)$ & $27(87.1 \%)$ & 0.916 & 0.932 & \\
\hline Metastases sites & & & 0.343 & 0.93 & \\
\hline Bone-only & $5(6.8 \%)$ & $3(9.7 \%)$ & - & - & \\
\hline CNS & $11(15.1 \%)$ & $8(25.8 \%)$ & - & - & \\
\hline Visceral & $57(78.1 \%)$ & $20(64.5 \%)$ & - & & \\
\hline ECOG & & & 0.711 & 0.93 & \\
\hline 0 & $21(40.4 \%)$ & $11(50.0 \%)$ & - & - & \\
\hline 1 & $24(46.2 \%)$ & $7(31.8 \%)$ & - & - & \\
\hline 2 & $3(5.8 \%)$ & 2 (9.1\%) & - & - & \\
\hline 3 & $4(7.7 \%)$ & $2(9.1 \%)$ & - & - & \\
\hline PD-L1 status & & & 0.277 & 0.93 & \\
\hline Negative & $7(9.5 \%)$ & $2(6.5 \%)$ & - & - & \\
\hline Positive & $5(6.8 \%)$ & $0(0.0 \%)$ & - & - & \\
\hline Not documented & $62(83.8 \%)$ & $29(93.5 \%)$ & - & - & \\
\hline TMB (RUO), muts/mB, Median (IQR) & $2.5(1.3,6.1)$ & $2.6(1.3,4.5)$ & 0.86 & 0.932 & \\
\hline MSI (RAW) & & & 0.491 & 0.93 & \\
\hline MSS & $55(74.3 \%)$ & $21(67.7 \%)$ & & & \\
\hline Not documented & $19(25.7 \%)$ & $10(32.3 \%)$ & & & \\
\hline Start Date, Median (Range) & $\begin{array}{l}2018-01-21 \\
(2012-02-14-2020-09-24)\end{array}$ & $\begin{array}{l}2017-09-18 \\
(2013-03-01-2020-6-09)\end{array}$ & 0.429 & 0.93 & \\
\hline Treatment Line & & & $3.23 e-08$ & 0 & * \\
\hline $1-2$ & $59(79.7 \%)$ & $7(22.6 \%)$ & & & \\
\hline $3+$ & $15(20.3 \%)$ & $24(77.4 \%)$ & & & \\
\hline Deceased & $47(63.5 \%)$ & $22(71.0 \%)$ & 0.463 & 0.93 & \\
\hline
\end{tabular}

*Denotes a statistically significant difference. 
type, ECOG, TMB, and treatment line using PSM. The TTNT on mTORi vs. chemotherapy was 6.5 months (95\% CI $4.6-\mathrm{n} / \mathrm{a}$ ) and 5.8 months (95\% CI 4.6 - na), respectively. The relative efficacy of receiving mTORi was not significantly higher than receiving chemo ( $\mathrm{HR}=0.8$ [95\% CI: $0.4-1.5], \mathrm{P}=0.5$ ).

After multidisciplinary discussion, the consensus recommendation was to start treatment with the combination of mTORi, everolimus, and AI, exemestane. Treatment was initiated on $8 / 28 / 20$, and the everolimus dose was decreased from 10 $\mathrm{mg}$ to $7.5 \mathrm{mg}$ daily due to the episode of neutropenia after the first cycle. Otherwise, the patient tolerated treatment without major side effects. By the second clinical visit in October 2020, the patient's breast was less erythematous, and the density of the tissue was lessening. There was no tenderness on palpation. PET/CT showed diminished left axillary uptake, decreased right paratracheal lymph node PET avidity, and stable bone disease consistent with a partial response. The most recent office visit in January 2021, breast exam revealed a normal-appearing skin with only faint erythema. All other skin lesions have resolved. The patient feels well and reports no pain.

\section{Discussion}

Roughly $10 \%$ of all breast cancers are invasive lobular carcinomas $(23,24)$. Invasive lobular carcinoma is strongly associated with exposure to female hormones, and its incidence is more subject to variation. It is more strongly associated with early menarche, late menopause, and late age of first birth. Of high-penetrance genes, BRCA1 and TP53 are predominantly associated with invasive ductal carcinoma (IDC), BRCA2 mutations are associated with both IDC and invasive lobular cancer (ILC), while mutations in CDH1 (encoding E-cadherin protein) are exclusively associated with ILC (25). It is characterized by functional loss of E-cadherin, resulting in cellularadhesion defect. Besides E-cadherin loss, Ciriello et al. identified mutations targeting PTEN, TBX3, and FOXA1 as ILC enriching features (26). PTEN loss is associ- ated with increased AKT phosphorylation, which was highest in ILC among all breast cancer subtypes. Spatially clustered FOXA1 mutations correlated with increased FOXA1 expression and activity. Conversely, GATA3mutations and high expression characterized Luminal A IDC, suggesting differential modulation of the ER activity in ILC and IDC. The proliferation and immune-related signatures determined three ILC transcriptional subtypes associated with survival differences. Mixed IDC/ILC cases were molecularly classified as ILC-like and IDC-like revealing no true hybrid features. This points to the heterogeneity of ILC and particularly to a distinct molecular profile of ILC vs. IDC. The case presented herein, however, seems to rather represent the Luminal B category, based on PR negative status and high Ki-67 (50\%) for ILC. Generally, ILC is considered as cancer with a good short-term prognosis. Metastatic ILC spreads more commonly to the ovaries, colon, omentum, and stomach. Interestingly, a high tumor mutational burden (TMB) is associated with metastatic ILC, with $8.9 \%$ of metastatic ILC classified as TMB-high (27).

The patient presented herein was treated with standard of care targeted therapy and aromatase inhibitors with some short-term success and ultimate progression. However, CGP opened another avenue of treatment that otherwise would not be one on the mind of treating physician. In addition, basic analysis of the CGP results did not directly point to the use of mTORi. The deeper up-and downstream analysis and discussion at our Molecular Tumor Board (MTB) uncovered those therapeutic options. An MTB also provides a unique setting for the application of RWD. During this MTB, RWD provided treatment information from patients in the CGDB that were genomically similar to the MTB patient. This, at least, at present, shows to be highly effective for this patient. When clinical literature for a biomarker is limited (such as $A K T 1$ ), the CGDB can provide clinical utility through decision support. Future studies should seek to understand which controls and confounders can improve clinical decision support using RWD. Our experience with MTB showed that al- 
most half of the patients (46\%) presented at MTB were offered genomically matched therapy or clinical trials (18). The patient presented here is one of $22 \%$ of patients who received recommended treatment. Others did not for different reasons, including physicians and patients' preferences, poor performance status, lack of coverage, etc. In the future, more extensive use of CGP, more wide availability of MTB's to treating physicians, wider accessibility to clinical trials, better education of physicians and community, and collaboration with third-party payers will open more possibilities for effective treatment of patients with advanced malignant diseases.

\section{Conclusion}

AKT1 mutations occur in $4 \%$ of breast cancer patients, and AKT1 E17K mutations account for $\sim 80 \%$ of those mutations (28). AKT1 E17K variants have been previously characterized as activating and oncogenic. The AKT1 E17K mutation is proposed to induce hyper activation of the mTOR pathway through constitutive AKT1 signaling and activation of the downstream components of the mTOR pathway $(29,30)$. Clinical study of mTOR pathway targeted therapy in AKT1 mutant breast cancer is still early in development. However, targeting AKT1 and downstream mTOR pathway components has shown efficacy in a limited number of AKT1 E17K mutant breast cancer patients $(28,31,32)$.

There is no consensus model for the application of comprehensive genomic profiling (CGP) and real-world data (RWD) in the treatment of cancer patients. This case study provides an example for the use of RWD and CGP in the context of a multidisciplinary Molecular Tumor Board (MTB). Furthermore, the study adds to the growing body of clinical literature that suggests that AKT1 mutant breast cancer patients may be sensitive to mTOR pathway targeted therapy in the advanced setting.

Authors' Contributions: Conception and design: HT, OH, and GS; Acquisition, analysis and interpretation of data: $\mathrm{OH}$, BT, JT, KS, KR, LZ, AM, MF, SW, MJF, and BA; Drafting the article: GS; Revising it critically for important intellectual content: HT and OH; Approved final version of the manuscript: HT, OH and GS.

Ethical (IRB) Approval: The case report was submitted to Sparrow IRB, and they have determined that this project does not meet the definition of human subject research under the purview of IRB according to federal regulations. Verbal approval of the patient was obtained by the treating physician to publish the case report.

Conflict of Interest: All authors have signed the journal's COI form. HT, BT, and JR have no conflict of interest in relation to this article. GS is on the Speaker's Bureau of Foundation Medicine, SW and MJF are consultants to Foundation Medicine; OH, KS, BA, AM, LZ, MF, and KR are employees of Foundation Medicine- Roche group.

\section{References}

1. Chen Y, Huang L, Dong Y, Tao C, Zhang R, Shao H, et al. Effect of AKT1 (p. E17K) Hotspot Mutation on Malignant Tumorigenesis and Prognosis. Front Cell Dev Biol [Internet]. 2020 [cited 2021 Jan 20]; 8. Available from: https:// www.frontiersin.org/articles/10.3389/fcell.2020.573599/ full\#B90.

2. Khatpe AS, Adebayo AK, Herodotou CA, Kumar B, Nakshatri H. Nexus between PI3K/AKT and Estrogen Receptor Signaling in Breast Cancer. Cancers. 2021; 13(3):369.

3. Hinz N, Jücker M. Distinct functions of AKT isoforms in breast cancer: a comprehensive review. Cell Commun Signal CCS. 2019;17(1):154.

4. Alwhaibi A, Verma A, Adil MS, Somanath PR. The unconventional role of Akt1 in the advanced cancers and in diabetes-promoted carcinogenesis. Pharmacol Res. 2019;145:104270.

5. Cole PA, Chu N, Salguero AL, Bae H. AKTivation mechanisms. Curr Opin Struct Biol. 2019;59:47-53.

6. Beaver JA, Gustin JP, Yi KH, Rajpurohit A, Thomas M, Gilbert SF, et al. PIK3CA and AKT1 Mutations Have Distinct Effects on Sensitivity to Targeted Pathway Inhibitors in an Isogenic Luminal Breast Cancer Model System. Clin Cancer Res. 2013;19(19):5413-22.

7. Davies BR, Guan N, Logie A, Crafter C, Hanson L, Jacobs V, et al. Tumors with AKT1E17K Mutations Are Rational Targets for Single Agent or Combination Therapy with AKT Inhibitors. Mol Cancer Ther. 2015;14(11):2441-51.

8. Marco CD, Malanga D, Rinaldo N, Vita FD, Scrima M, Lovisa S, et al. Mutant AKT1- E17K is oncogenic in lung epithelial cells. Oncotarget. 2015;6(37):39634-50.

9. Oeck S, Al-Refae K, Riffkin H, Wiel G, Handrick R, Klein D, et al. Activating Akt1 mutations alter DNA double strand break repair and radiosensitivity. Sci Rep. 2017;7(1):42700. 
10. Van Der Zwet JCG, Buijs-Gladdines JGCAM, Mollova I, Graus L, Bos FL, Rios AC, et al. The Clinical and Functional Relevance of the Transforming AKT E17K Mutation in T-ALL. Blood. 2019;134(Suppl 1):3804.

11. Wu W, Chen Y, Huang L, Li W, Tao C, Shen H. Effects of AKT1 E17K mutation hotspots on the biological behavior of breast cancer cells. Int J Clin Exp Pathol. 2020;13(3):332-46.

12. Elghazi L, Balcazar N, Bernal-Mizrachi E. Emerging role of protein kinase $\mathrm{B} /$ Akt signaling in pancreatic $\beta$-cell mass and function. Int J Biochem Cell Biol. 2006;38(2):157-63.

13. Hechtman JF, Sadowska J, Huse JT, Borsu L, Yaeger R, Shia J, et al. AKT1 E17K in Colorectal Carcinoma Is Associated with BRAF V600E but Not MSI-H Status: A Clinicopathologic Comparison to PIK3CA Helical and Kinase Domain Mutants. Mol Cancer Res. 2015;13(6):1003-8.

14. Hyman DM, Smyth LM, Donoghue MTA, Westin SN, Bedard PL, Dean EJ, et al. AKT Inhibition in Solid Tumors With AKT1 Mutations. J Clin Oncol. 2017;35(20):2251-9.

15. Smyth LM, Tamura K, Oliveira M, Ciruelos EM, Mayer IA, Sablin M-P, et al. Capivasertib, an AKT Kinase Inhibitor, as Monotherapy or in Combination with Fulvestrant in Patients with AKT1E17K-Mutant, ER-Positive Metastatic Breast Cancer. Clin Cancer Res. 2020;26(15):394757.

16. Smyth LM, Zhou Q, Nguyen B, Yu C, Lepisto EM, Arnedos $\mathrm{M}$, et al. Characteristics and Outcome of AKT1E17KMutant Breast Cancer Defined through AACR Project GENIE, a Clinicogenomic Registry. Cancer Discov. 2020;10(4):526-35.

17. Tamura K, Hashimoto J, Tanabe Y, Kodaira M, Yonemori K, Seto T, et al. Safety and tolerability of AZD5363 in Japanese patients with advanced solid tumors. Cancer Chemother Pharmacol. 2016;77(4):787-95.

18. Trivedi H, Acharya D, Chamarthy U, Meunier J, AliAhmad H, Hamdan M, et al. Implementation and Outcomes of a Molecular Tumor Board at Herbert-Herman Cancer Center, Sparrow Hospital. Acta Med Acad. 2019;48(1):105-15.

19. Singal G, Miller PG, Agarwala V, Li G, Kaushik G, Backenroth D, et al. Association of Patient Characteristics and Tumor Genomics With Clinical Outcomes Among Patients With Non-Small Cell Lung Cancer Using a Clinicogenomic Database. JAMA. 2019;321(14):1391-9.

20. Madison R, Schrock AB, Castellanos E, Gregg JP, Snider J, Ali SM, et al. Retrospective analysis of real-world data to determine clinical outcomes of patients with advanced non- small cell lung cancer following cell-free circulating tumor DNA genomic profiling. Lung Cancer Amst Neth. 2020;148:69-78.

21. Schrock AB, Lee JK, Sandhu J, Madison R, Cho-Phan C, Snider JW, et al. RAS amplification as a negative predictor of benefit from anti-EGFR containing therapy regimens in metastatic colorectal cancer. The Oncologist. 2021 January 19. Online ahead of print.

22. Yarchoan M, Albacker LA, Hopkins AC, Montesion M, Murugesan K, Vithayathil TT, et al. PD-L1 expression and tumor mutational burden are independent biomarkers in most cancers. JCI Insight [Internet]. 2019 Mar 21 [cited 2021 Feb 11];4(6). Available from: https://insight.jci.org/ articles/view/126908.

23. Li CI, Anderson BO, Daling JR, Moe RE. Trends in incidence rates of invasive lobular and ductal breast carcinoma. JAMA. 2003;289(11):1421-4.

24. Li CI, Daling JR. Changes in breast cancer incidence rates in the United States by histologic subtype and race/ ethnicity, 1995 to 2004. Cancer Epidemiol Biomark Prev Publ Am Assoc Cancer Res Cosponsored Am Soc Prev Oncol. 2007;16(12):2773-80.

25. Dossus L, Benusiglio PR. Lobular breast cancer: incidence and genetic and non-genetic risk factors. Breast Cancer Res BCR. 2015;17:37.

26. Ciriello G, Gatza ML, Beck AH, Wilkerson MD, Rhie SK, Pastore A, et al. Comprehensive Molecular Portraits of Invasive Lobular Breast Cancer. Cell. 2015;163(2):506-19.

27. Sokol ES, Basudan A, Lee AV, Stephens PJ, Frampton GM, Oesterreich S, et al. Abstract PD8-05: Genomic profiling of metastatic invasive lobular carcinoma reveals unique genomics and therapeutic opportunities. Cancer Res. 2018;78(4 Suppl):PD8-05-PD8-05.

28. Tamura K, Hashimoto J, Tanabe Y, Kodaira M, Yonemori K, Seto T, et al. Safety and tolerability of AZD5363 in Japanese patients with advanced solid tumors. Cancer Chemother Pharmacol. 2016;77(4):787-95.

29. Chen Y, Huang L, Dong Y, Tao C, Zhang R, Shao H, et al. Effect of AKT1 (p. E17K) Hotspot Mutation on Malignant Tumorigenesis and Prognosis. Front Cell Dev Biol. 2020;8:573599. doi: 10.3389/fcell.2020.573599.

30. Wu W, Chen Y, Huang L, Li W, Tao C, Shen H. Effects of AKT1 E17K mutation hotspots on the biological behavior of breast cancer cells. Int J Clin Exp Pathol. 2020;13(3):332-46.

31. Smyth LM, Tamura K, Oliveira M, Ciruelos EM, Mayer IA, Sablin M-P, et al. Capivasertib, an AKT Kinase Inhibitor, as Monotherapy or in Combination with Fulvestrant in Patients with AKT1E17K-Mutant, ER-Positive Metastatic Breast Cancer. Clin Cancer Res. 2020;26(15):394757.

32. Smyth LM, Zhou Q, Nguyen B, Yu C, Lepisto EM, Arnedos M, et al. Characteristics and Outcome of AKT1E17KMutant Breast Cancer Defined through AACR Project GENIE, a Clinicogenomic Registry. Cancer Discov. 2020;10(4):526-35. (Smyth 2020b) 